ARTICLE

https://doi.org/10.1038/s41467-019-13953-1

\title{
Dopamine transients do not act as model-free prediction errors during associative learning
}

\author{
Melissa J. Sharpe (1) 1,2,3,4, Hannah M. Batchelor ${ }^{1}$, Lauren E. Mueller', Chun Yun Chang ${ }^{1}$, Etienne J.P. Maes (1) 1,
} Yael Niv (1) ${ }^{2,5}$ \& Geoffrey Schoenbaum (1) $1,6,7 \star$

Dopamine neurons are proposed to signal the reward prediction error in model-free reinforcement learning algorithms. This term represents the unpredicted or 'excess' value of the rewarding event, value that is then added to the intrinsic value of any antecedent cues, contexts or events. To support this proposal, proponents cite evidence that artificiallyinduced dopamine transients cause lasting changes in behavior. Yet these studies do not generally assess learning under conditions where an endogenous prediction error would occur. Here, to address this, we conducted three experiments where we optogenetically activated dopamine neurons while rats were learning associative relationships, both with and without reward. In each experiment, the antecedent cues failed to acquire value and instead entered into associations with the later events, whether valueless cues or valued rewards. These results show that in learning situations appropriate for the appearance of a prediction error, dopamine transients support associative, rather than model-free, learning.

\footnotetext{
${ }^{1}$ National Institute on Drug Abuse, Intramural Research Program, Baltimore, MD 21224, USA. ${ }^{2}$ Princeton Neuroscience Institute, Princeton University, Princeton, NJ 08544, USA. ${ }^{3}$ School of Psychology, UNSW, Australia. ${ }^{4}$ Department of Psychology, University of California, Los Angeles, CA 90095-1563, USA. ${ }^{5}$ Psychology Department, Princeton University, Princeton, NJ 08544, USA. ${ }^{6}$ Departments of Anatomy \& Neurobiology and Psychiatry, University of Maryland School of Medicine, Baltimore, MD 21201, USA. ${ }^{7}$ Solomon H. Snyder Department of Neuroscience, The Johns Hopkins University, Baltimore, MD 21287, USA. *email: geoffrey.schoenbaum@nih.gov
} 
$\mathrm{D}$ opamine neurons have been famously shown to fire to unexpected rewards ${ }^{1}$. The most popular idea in the field is that these transient bursts of activity (and the brief pauses on omission of an expected reward) act as the prediction error described in model-free reinforcement learning algorithms ${ }^{2}$. This error term captures the difference between the actual and expected value of the rewarding event and then assigns this 'excess' value to any antecedent cue, context, or event ${ }^{3}$. Critically, such 'cached' or learned values are separate from associative or model-based representations linking the actual external events to one another ${ }^{4}$. This proposal is supported mainly by correlative studies (see ref. ${ }^{5}$, for full review). However, support also comes from a growing number of causal reports, showing that artificially induced dopamine transients, with a brevity and timing similar to the physiological prediction-error correlates, are able to drive enduring changes in behavior to antecedent cues, contexts, or events ${ }^{6-13}$. These changes are assumed to reflect value learning, however almost none of these studies investigate the informational content of the learning to confirm or refute this assumption.

Two studies stand as exceptions to this general statement ${ }^{6,8}$. In both, artificially induced dopamine transients, constrained within blocking paradigms to eliminate naturally occurring teaching signals, were shown to cause associations to form between external events. In one study, the association was formed between a cue and the sensory properties of the reward ${ }^{8}$, and in the other, the association was formed between a cue and the sensory properties of another cue ${ }^{6}$. Critically, in each experiment, the learning driven by the artificial transient was similar to the learning that would have been observed in the absence of blocking. However, while these results cannot be explained by cached-value learning mechanisms, neither of these studies included an assessment of value that was independent of the associative prediction of reward. Thus, these studies did not directly address whether the dopamine transient acted only to facilitate associative learning or also promoted model-free valuecaching.

In fact, to the best of our knowledge, there is currently no report that directly tests whether a transient increase in the activity of midbrain dopamine neurons functions to assign value directly to cues when it is delivered in contexts where a prediction error might normally be expected to appear during associative learning. While exceptions exist, these contexts commonly have at least two events arranged in a relatively precise relationship to the error signal-one whose onset co-occurs with the onset of the error and another whose onset is antecedent to the error. Testing the effect of artificially induced dopamine transients within such a setting is potentially important since prediction errors delivered outside of such constraints may act in ways that are outside of their normal function. Here we conducted three experiments designed to place a dopamine transient in a learning context defined by two external cues with the above relationship. We used conditioned reinforcement to assess cue value independent of the associative predictions about reward, and in each experiment we found that the dopamine transient supported the development of associative representations without endowing the antecedent cues with value.

\section{Results}

Dopamine can unblock learning without adding value. Prior to training, all rats underwent surgery to infuse virus and implant fiber optics targeting the ventral tegmental area (VTA; Fig. 1). We infused AAV5-EF1 $\alpha$-DIO-ChR2-eYFP (channelrhodopsin-2 (ChR2) experimental group; $n=8$ ) or AAV5-EF1 $\alpha$-DIO-eYFP (eYFP control group, $n=8$ ) into the VTA of rats expressing Cre
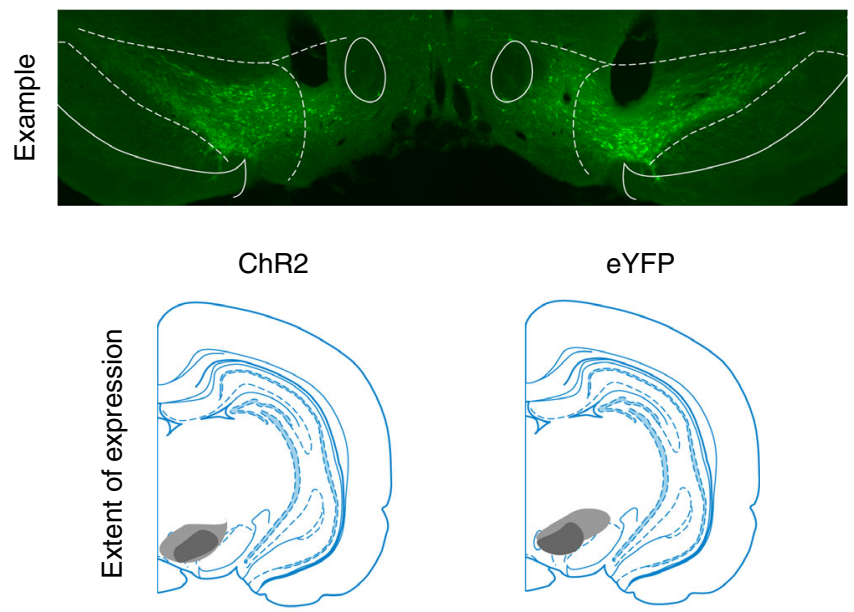

eYFP
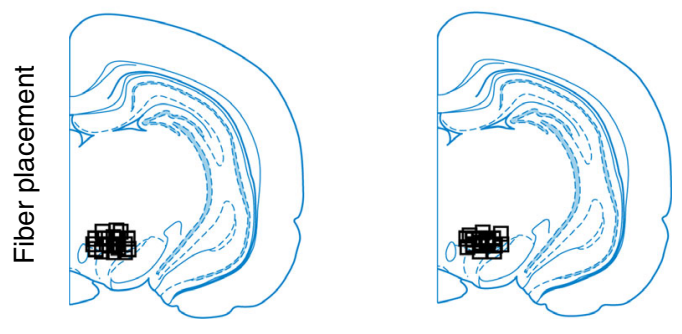

Fig. 1 Histological verification of Cre-dependent ChR2 and eYFP in TH+ neurons and fiber placement in the VTA for Experiments 1, 2, and 3. Top row: example of the extent and location of virus expression in VTA and openings in the tissue reflecting fiber placement, $\sim 5 \mathrm{~mm}$ posterior to bregma. Middle row: Representation of the bilateral viral expression; dark shading represents the minimal and the light shading the maximal spread of expression at the center of injection sites. Bottom row: Approximate location of fiber tips in VTA, indicated by black squares.

recombinase under the control of the tyrosine hydroxylase $(\mathrm{TH})$ promoter ${ }^{14}$. After surgery and recovery, rats were food deprived and then trained on the blocking of sensory preconditioning task (Fig. 2) used previously ${ }^{6}$. The use of a design that isolates dopamine transients when rats are learning about neutral information is advantageous because it dissociates the endogeonous value signal elicited by a motivationally-significant reward (e.g., food) from any value that may be inherent in the dopamine transient itself. Thus, it provides an ideal test for determining whether dopamine tranisents endow cues with value.

Training began with 2 days of preconditioning. On the first day, the rats received 16 pairings of two 10-s neutral cues $(A \rightarrow X)$. On the second day, the rats continued to receive pairings of the same two neutral cues ( $\mathrm{A} \rightarrow \mathrm{X} ; 8$ trials). In addition, on other trials, the first cue, $\mathrm{A}$, was presented together with a second, novel neutral cue, still followed by $\mathrm{X}$ (either $\mathrm{AC} \rightarrow \mathrm{X}$ or $\mathrm{AD} \rightarrow \mathrm{X}$; eight trials each). Because A predicts $\mathrm{X}$, this design causes acquisition of the $\mathrm{C} \rightarrow \mathrm{X}$ and $\mathrm{D} \rightarrow \mathrm{X}$ associations to be blocked. On $\mathrm{AC}$ trials, blue light $(473 \mathrm{~nm}, 20 \mathrm{~Hz}, 16-18 \mathrm{~mW}$ output; Shanghai Laser \& Optics Century Co., Ltd) was delivered for $1 \mathrm{~s}$ at the start of X to activate VTA dopamine neurons, giving the transient an opportunity to both assign value to cue $\mathrm{C}$ and also to unblock acquisition of the $\mathrm{C} \rightarrow \mathrm{X}$ association. As a temporal control for nonspecific effects, the same light pattern was delivered in the inter-trial interval on AD trials, $120-180 \mathrm{~s}$ after termination of X. Finally, as a positive control for normal learning, the rats also received pairings of two novel 10 -s cues with $\mathrm{X}(\mathrm{EF} \rightarrow \mathrm{X}$; eight trials). Rats exhibited little responding at the food port on either 


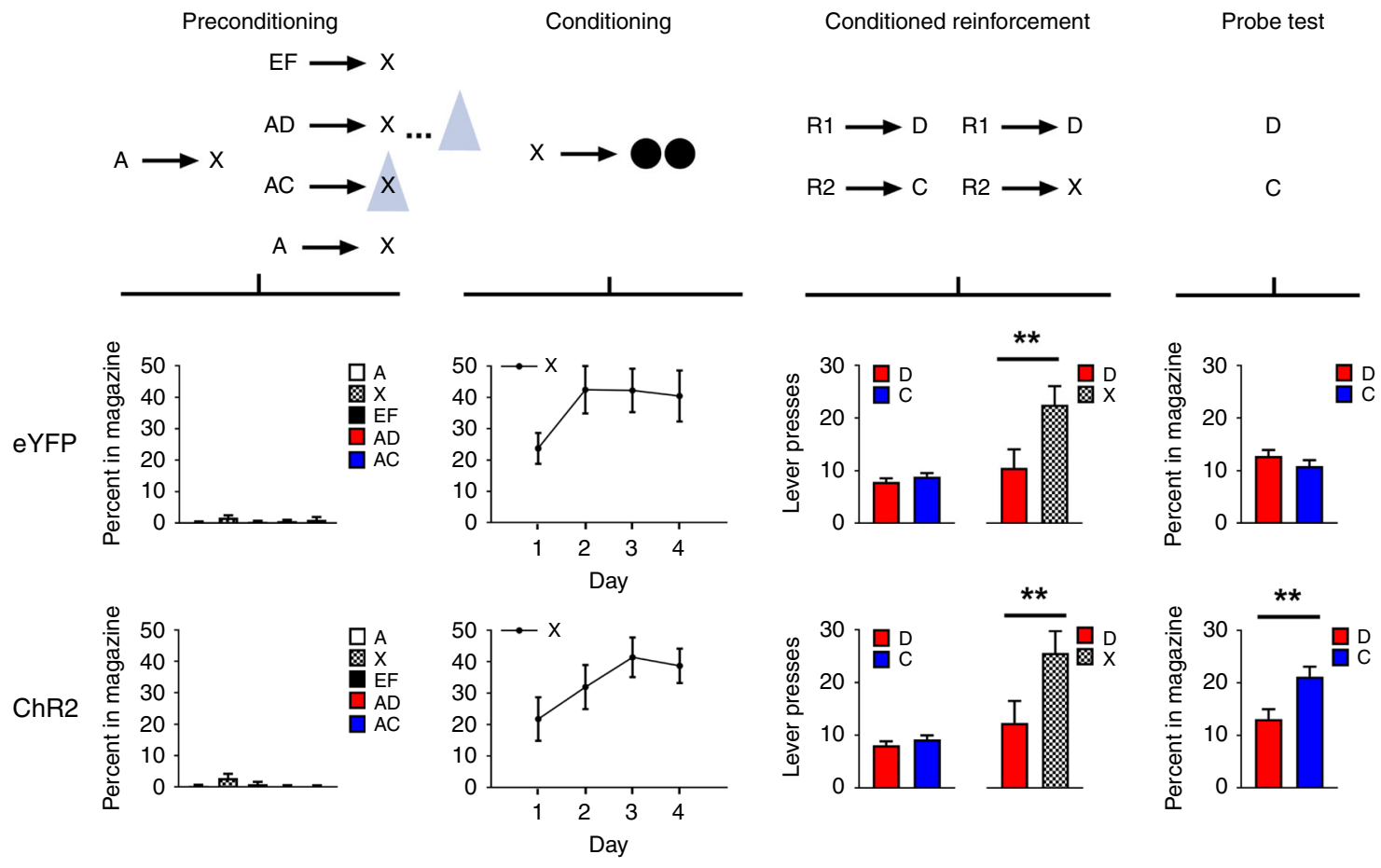

Fig. 2 Brief optogenetic stimulation of dopamine neurons in the VTA produces learned associations without endowing cues with cached value. Top row: Schematic illustrating the task design, which consisted of preconditioning and conditioning, followed by conditioned reinforcement and probe testing. $A$ and $E$ indicate visual cues, while C, D, F and X indicate auditory cues. R1 and R2 indicate differently positioned levers, and black circles show delivery of food pellets. VTA dopamine neurons were activated by light delivery in our ChR2 experiment group $(n=8)$ but not in our eYFP control group ( $n=8$ ), illustrated by the blue triangle, for $1 \mathrm{~s}$ at the beginning of $\mathrm{X}$ on $\mathrm{AC}$ trials and in the inter-trial interval on AD trials. Middle and bottom rows: Plots show rates of responding (with standard error of the mean represented above and below the mean; \pm SEM) across each phase of training, aligned to the above schematic. The top row of plots show data for the eYFP control group and bottom row of plots show data for the ChR2 experimental group. For individual rats' responses see Supplementary Fig. 3.

day of training (Fig. 2, preconditioning), with a two-factor ANOVA on the percent of time spent in the food port during cue presentation revealing no significant effects involving group (group: $F_{1,14}=0.027, p=0.873$; cue $\times$ group: $F_{4,56}=0.509, p=$ $0.729)$. An analysis of responding during the pre-cue period using a one-way ANOVA also did not reveal any group differences [mean(SEM)\%: ChR2 1.36 (0.58), eYFP $2.14(0.62) ; F_{1,14}=0.823$, $p=0.380]$.

Following preconditioning, the rats underwent 4 days of conditioning to associate $\mathrm{X}$ with food reward. Each day, the rats received 24 trials in which $\mathrm{X}$ was presented, followed by delivery of two $45-\mathrm{mg}$ sucrose pellets ( $\rightarrow 2 \mathrm{US}$ ). Rats in both groups acquired a conditioned response, increasing the amount of time spent in the food port during presentation of cue $\mathrm{X}$ with no difference in either the level of this response or in the rate at which it was acquired in the two groups (Fig. 2, conditioning). A two-factor ANOVA (day $\times$ group) revealed only a main effect of day (day: $F_{3,42}=8.258, p=0.000$; group: $F_{1,14}=0.256, p=0.621$; day x group: $F_{3,42}=0.513, p=0.675$ ). Additionally, an analysis of responding during the pre-cue period using a one-way ANOVA did not reveal any group differences [mean(SEM)\%: ChR2 6.81 (1.23), eYFP 5.84 (1.31); $\left.F_{1,14}=0.278, p=0.606\right]$.

After conditioning, we assessed whether the preconditioned cue $\mathrm{C}$ had acquired any value by virtue of its pairing with a dopamine transient. To do this, we measured the ability of $\mathrm{C}$ to promote conditioned reinforcement, which is an iconic test typically used to assess the value of a cue after pairing with a natural reward or drug of abuse ${ }^{15}$. Critically, conditioned reinforcement is generally accepted as reflecting the value of the cue, independent of any expectations of reward delivery, since it is not normally affected by devaluation of the predicted reward ${ }^{16}$. Thus, conditioned reinforcement is a good measure of acquired value independent of other learning. In our conditioned reinforcement test, we measured lever pressing during a 30 minute session in which two levers were made available in the experimental chamber, one that led to a $2 \mathrm{~s}$ presentation of $\mathrm{D}$ and another that led to a $2 \mathrm{~s}$ presentation of $\mathrm{C}(\mathrm{R} 1 \rightarrow \mathrm{D}, \mathrm{R} 2 \rightarrow \mathrm{C})$. In addition, as a positive control for conditioned reinforcement, the same rats also received a second conditioned reinforcement test in which two levers were again made available in the experimental chamber, this time leading to presentation of either $\mathrm{D}$ or $\mathrm{X}$ $(\mathrm{R} 1 \rightarrow \mathrm{D}, \mathrm{R} 2 \rightarrow \mathrm{X})$. Because $\mathrm{X}$ had been directly paired with reward, it should have acquired value that would support conditioned reinforcement ${ }^{16-18}$.

The results of the conditioned reinforcement test showed that the dopamine transient did not endow the preconditioned cue $\mathrm{C}$ with value; rats in the ChR2 group pressed at the same low rates for both $\mathrm{C}$ and $\mathrm{D}$ as did eYFP controls (Fig. 2, conditioned reinforcement). A two-factor ANOVA (cue $\times$ group) revealed no significant effects (cue: $F_{1,14}=1.094, p=0.313$; group: $F_{1,14}=$ $0.006, p=0.940$; cue $\times$ group: $F_{1,14}=0.004, p=0.952$ ). Importantly, the failure to observe conditioned reinforcement for $\mathrm{C}$ was not because of any deficiency in conditioned reinforcement, since the ChR2 rats showed conditioned reinforcement equivalent to that seen eYFP rats when they were given a chance to lever press for $\mathrm{X}$, which had acquired value through direct pairing with reward (Fig. 2, conditioned reinforcement). A two-factor ANOVA (cue $\times$ group) revealed a significant main effect of cue 
$\left(F_{1,14}=5.297, p=0.037\right)$ without any effects of group (group: $F_{1,14}=0.120, p=0.734$; interaction: $\left.F_{1,14}=0.014, p=0.907\right)$. Parenthetically, the absence of any group effects suggests that the dopamine transient also failed to add to the value that $\mathrm{X}$ acquired by its pairing with reward; this is also suggested by the similar learning rates supported by $\mathrm{X}$ during conditioning.

Finally, to confirm that the dopamine transient was effective in producing learning between $\mathrm{C} \rightarrow \mathrm{X}$, we assessed the Pavlovian response elicited by the preconditioned cues. To do this, rats received a probe test in which preconditioned cues $\mathrm{D}$ and $\mathrm{C}$ were presented, alone and without reward, and we measured how much time the rats spent in the food port. Unlike conditioned reinforcement, food port responding in this context is a measure shown previously to reflect a specific expectation of reward delivery, since it is sensitive to devaluation of the predicted reward $^{6}$. The results of the probe test confirmed that the dopamine transient unblocked learning in the preconditioning phase; rats in the ChR2 group showed a significant increase in the time spent in the food port during presentation of $\mathrm{C}$ relative to $\mathrm{D}$, a difference that was not seen in the eYFP group (Fig. 2, probe test). A two-factor ANOVA (cue $\times$ group) revealed a significant interaction $\left(F_{1,14}=5.060, p=0.041\right)$, due to a significant difference between cues $\mathrm{C}$ and $\mathrm{D}$ in the ChR2 group $\left(F_{1,14}=\right.$ 6.578, $p=0.022)$ but not in the eYFP control group $\left(F_{1,14}=\right.$ $0.380, p=0.547)$. Again, an analysis of responding during the pre-cue period using a one-way ANOVA did not reveal any group differences [mean(SEM)\%: ChR2 0.14 (0.04), eYFP 0.27 (0.08); $\left.F_{1,14}=2.491, p=0.137\right]$. Thus, the introduction of a dopamine transient at the beginning of $\mathrm{X}$ on $\mathrm{AC}$ trials was sufficient to selectively unblock learning about the $\mathrm{C}-\mathrm{X}$ association (refer to Supplementary Fig. 1 for analyses of this effect with number of entries into magazine $)^{6}$, but did not endow cue $\mathrm{C}$ with model-free value. Importantly, we subsequently showed that rats will press a lever to receive stimulation of these same dopamine neurons (Supplementary Fig. 2).

Dopamine can accelerate overlearning without adding value. The above experiment demonstrates that a dopamine transient does not endow a cue with value, as indexed by conditioned reinforcement, despite driving the formation of an associative model of events, revealed by the increase in food cup responding to the same cue. This result is contrary to predictions of the hypothesis that the dopamine transient functions as the prediction error described in model-free reinforcement learning algorithms. However, this design could be viewed as a weak test of this hypothesis, since dopamine neurons were only activated a few times, and both hypotheses generally predict more responding. To address these shortcomings, we designed a more stringent test in which the dopamine neurons were activated many more times, and the predictions of the two accounts were placed into opposition.

To do to this, we returned to the standard sensory preconditioning task $\mathrm{k}^{19}$. Sensory preconditioning is a particularly interesting task to use for this purpose, since responding in the probe test is known to be sensitive to the number of pairings of the cues in the initial preconditioning phase $(A \rightarrow B)$, increasing with several pairings but then declining thereafter ${ }^{20-23}$. We observed this effect ourselves, when we exposed rats to 48 pairings of $\mathrm{A} \rightarrow \mathrm{B}$ during preconditioning (Supplementary Fig. 3), instead of the usual $12^{6,18,24,25}$. Diminished responding in the probe test with increasing experience in the preconditioning phase is thought to reflect a stronger association between cues $\mathrm{A}$ and $\mathrm{B}$, which causes "B-after- $\mathrm{A}$ " to become dissociated from "Balone" 22 . As a result of this, the association between $B$ and reward, acquired in conditioning, does not generalize to the representation of $\mathrm{B}$ evoked by the preconditioned cue, $\mathrm{A}^{20-23}$. This phenomenon leads to a somewhat counterintuitive prediction, which is that if the dopamine transient applied in the first experiment is mimicking the normal teaching signal, then delivering it in the standard preconditioning design should diminish evidence of preconditioning, due to an acceleration of this overlearning effect. Of course, this would be in opposition to the value hypothesis, which would simply predict more responding with more value.

Prior to training, all rats underwent surgery to infuse virus and implant fiber optics targeting the VTA as in Experiment 1 (Fig. 1; ChR2 experimental group, $n=14$; eYFP control group, $n=14$ ). After surgery and recovery, rats were food deprived and then trained on the task. This began with one day of preconditioning, in which the rats received 12 presentations each of two pairs of 10 -s neutral cues (i.e., $\mathrm{A} \rightarrow \mathrm{B}$; $\mathrm{C} \rightarrow \mathrm{D}$ ). On $\mathrm{A} \rightarrow \mathrm{B}$ trials, blue light (473 nm, $20 \mathrm{~Hz}, 16-18 \mathrm{~mW}$ output; Shanghai Laser \& Optics Century Co., Ltd) was delivered for $1 \mathrm{~s}$ at the beginning of cue B to activate VTA dopamine neurons. Again, rats exhibited little responding at the food port (Fig. 3, preconditioning), with a twofactor ANOVA on the percent of time spent in the food port during cue presentation revealing no significant effects involving group (group: $F_{1,26}=0.047, p=0.831$ : cue $\times$ group: $F_{3,78}=0.470$, $p=0.704)$. An analysis of responding during the pre-cue period using a one-way ANOVA did not reveal any group differences [mean(SEM)\%: ChR2 7.03 (3.34), eYFP 5.43 (1.71); $F_{1,26}=0.183$, $p=0.672]$.

Following preconditioning, rats began conditioning, which continued for 4 days. Each day, the rats received 12 trials in which B was presented, followed by delivery of two 45-mg sucrose pellets $(\mathrm{B} \rightarrow 2 \mathrm{US}$ ), and 12 trials in which $\mathrm{D}$ was presented without reward $(\mathrm{D} \rightarrow$ nothing). Rats in both groups acquired a conditioned response, increasing the amount of time spent in the food port during cue presentation, and this response came to discriminate between the two cues as conditioning progressed (Fig. 3, conditioning). Importantly there was no difference in the level of conditioned responding to $\mathrm{B}$ and $\mathrm{D}$ across the two groups, or in the rate at which conditioned responding was acquired. Accordingly, a three-factor ANOVA (cue $\times$ day $\times$ group) revealed main effects of cue (i.e., B vs $\mathrm{D} ; F_{1,26}=37.371, p=0.000$ ) and day $\left(F_{3,78}=7.777, p=0.000\right)$ and a significant day $\times$ cue interaction $\left(F_{3,78}=30.293, p=0.000\right)$, but no significant effects involving group (group: $F_{1,26}=0.041, p=0.841$; cue $\times$ group: $F_{1,26}=0.219$, $p=0.643$; day $\times$ group: $F_{3,78}=0.565, \quad p=0.640 ;$ cue $\times$ day $\times$ group: $\left.F_{3,78}=1.028, p=0.385\right)$. An analysis of responding during the pre-cue period using a one-way ANOVA also did not reveal any group differences [mean(SEM)\%: ChR2 7.61 (1.68), eYFP 6.12 (0.94); $F_{1,26}=0.601, p=0.445$ ].

Rats then underwent conditioned reinforcement testing $(\mathrm{R} 1 \rightarrow \mathrm{A}$ vs $\mathrm{R} 2 \rightarrow \mathrm{C}$; $\mathrm{R} 1 \rightarrow \mathrm{B}$ vs $\mathrm{R} 2 \rightarrow \mathrm{D})$ to assess whether value had accrued to the preconditioned cues. This testing showed that simplifying the preconditioning design and increasing the number of pairings with the dopamine transient was still insufficient to cause acquisition of value by the preconditioned cues. Rats in the ChR2 group lever-pressed at the same low rates for both $\mathrm{A}$ and $\mathrm{C}$ as controls (Fig. 3, conditioned reinforcement), and a two-factor ANOVA (cue $\times$ group) revealed no significant effects (cue: A vs. C; $F_{1,26}=0.29, p=0.867$; group: $F_{1,26}=0.634$, $p=0.433$; cue $\times$ group: $F_{1,26}=0.019, p=0.892$ ). By contrast, rats in both groups showed normal conditioned reinforcement when given a chance to lever press for cue $B$, which had acquired value through direct pairing with reward (Fig. 3, conditioned reinforcement). A two-factor ANOVA (cue $\times$ group) revealed a significant main effect of cue $\left(F_{1,26}=7.132, p=0.013\right)$ without any group effects (group: $F_{1,26}=0.933, p=0.343$; cue $\times$ group: $\left.F_{1,26}=0.183, p=0.672\right)$. 

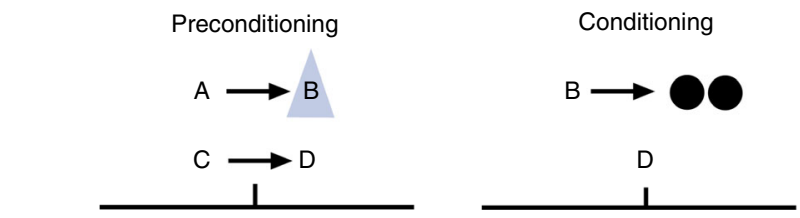

Conditioned reinforcement
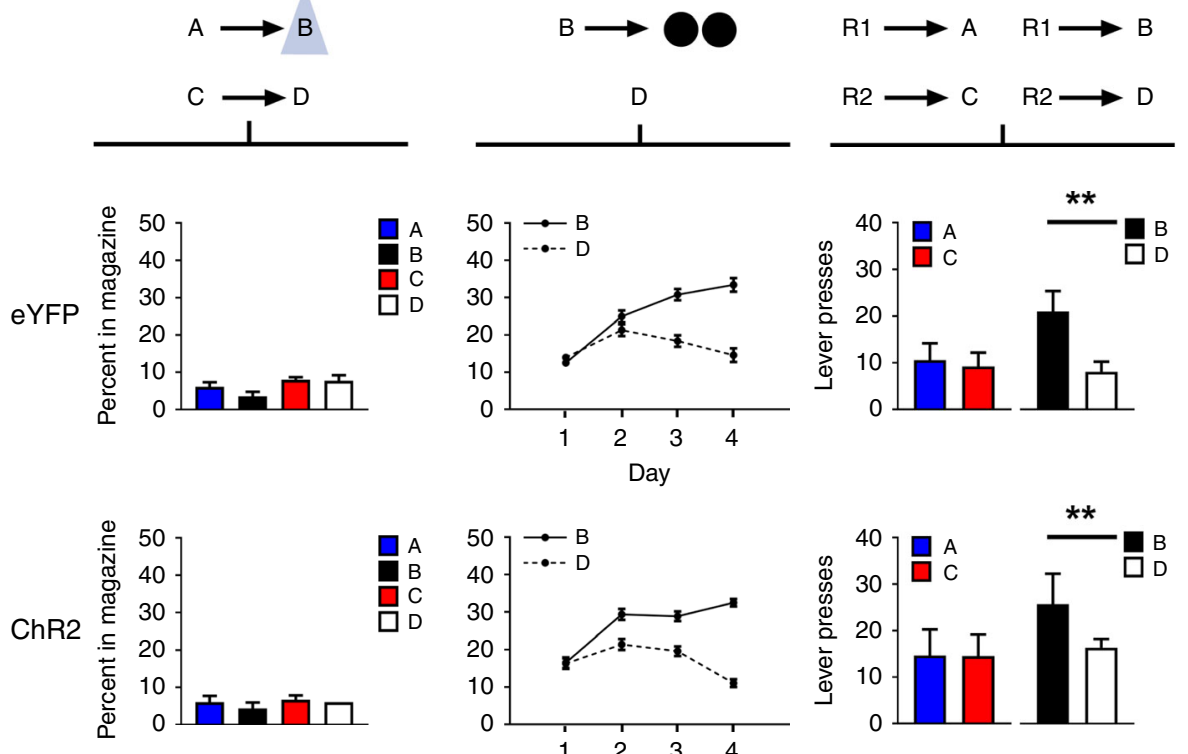
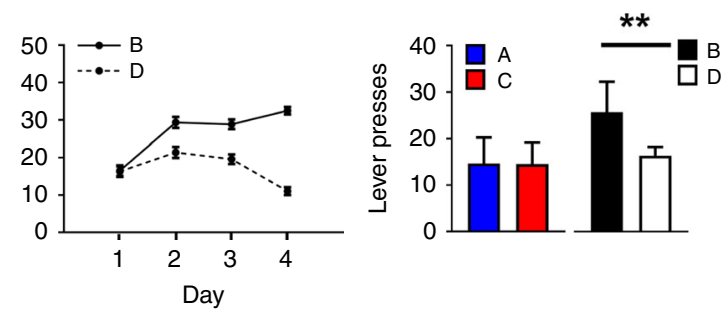

Probe test
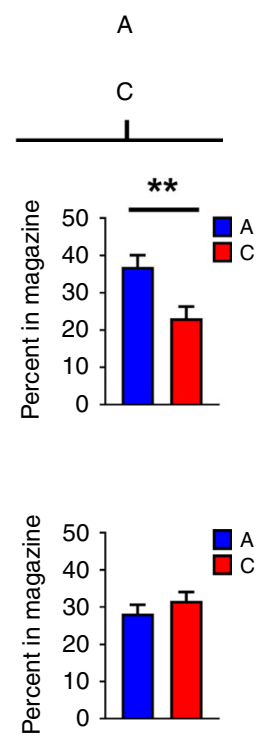

Fig. 3 Brief optogenetic stimulation of dopamine neurons in the VTA impairs the sensory preconditioning effect without endowing cues with cached value. Top row: Schematic illustrating the task design, which consisted of preconditioning and conditioning, followed by conditioned reinforcement and probe testing. A, B, C, and D indicate auditory cues, R1 and R2 indicate differently positioned levers, and black circles show delivery of food pellets. VTA dopamine neurons were activated by light delivery, illustrated by the blue triangle, at the beginning of B in our ChR2 group ( $n=14$; bottom plots), but not in our eYFP control group $(n=14$; top plots). Plots show rates of responding ( \pm SEM) across each phase of training, aligned to the above schematic. For individual rats' responses, see Supplementary Fig. 4.

Finally, rats received a probe test in which we presented cues A and $\mathrm{C}$, alone and without reward. If the dopamine transient in our design functions as a normal teaching signal, then we should see a reduction or loss of food port responding in the probe test, since overlearning of the preconditioned cue pairs normally has this effect (Supplementary Fig. 4). Consistent with this prediction, rats in the ChR2 group failed to show the normal difference in food port responding during presentation of $\mathrm{A}$ and $\mathrm{C}$ in the probe test (Fig. 3, probe test). A two-factor ANOVA (cue $\times$ group) revealed a significant interaction $\left(F_{1,26}=4.783, p=0.038\right)$, due to a difference in responding to $\mathrm{A}$ and $\mathrm{C}$ in the eYFP group $\left(F_{1,26}=\right.$ $6.113, p=0.020)$ that was not present in the ChR2 group $\left(F_{1,26}=\right.$ $0.385, p=0.540)$. An analysis of responding during the pre-cue period using a one-way ANOVA did not reveal any group differences [mean(SEM)\%: ChR2 5.46 (1.22), eYFP 5.15 (01.38); $\left.F_{1,26}=0.032, p=0.860\right]$.

These findings again show that a dopamine transient is not normally acting as a prediction error that trains cached values; rats would not work to obtain the cue that was paired with the transient. In this experiment, the absence of conditioned reinforcement was observed despite a larger number of presentations of the transient and in the absence of any potential interference due to food predictions that were evident for this cue in the first experiment. Here it is worth emphasizing that while the above results might be hard to square with the cached value hypothesis, they are precisely what would be predicted for associative, model-based learning. That is, sensory preconditioning normally produces a cue that supports model-based fooddirected responding but not conditioned reinforcement, and this responding is sensitive to overtraining. Thus in the above two experiments, the artificial induction of a dopamine transient seemingly supports valueless associative learning rather than cached-value learning.
Dopamine can drive configural learning without adding value. Finally, we tested whether our findings would generalize outside of learning about neutral sensory cues. For this, we used a configural learning task that allowed us to introduce dopamine transients in rats learning about cues and rewards, but in a way that would still allow us to dissociate value-caching from associative learning. On some trials, rats were presented with one of two auditory cues, one predicting reward and one predicting no reward (i.e., $\mathrm{A} \rightarrow$ nothing; $\mathrm{B} \rightarrow 2 \mathrm{US}$ ). On other trials, these same cues were preceeded by a common visual cue, $\mathrm{X}$, and the reward contingencies were switched (i.e. $\mathrm{X} \rightarrow \mathrm{A} \rightarrow 2 \mathrm{US}$; $\mathrm{X} \rightarrow \mathrm{B} \rightarrow$ nothing). We delivered a dopamine transient at the onset of $\mathrm{A}$ and $\mathrm{B}$, only when these cues were preceeded by $\mathrm{X}$. To learn this task, rats must distinguish "X-A" and "X-B" from "A-alone" and "B-alone", representations similar to those thought to underlie the overlearning effect in sensory preconditioning (Supplementary Fig. 4). We reasoned that if dopamine transients were facilitating overlearning in the second experiment by encouraging the formation of such configural representations, then a similar manipulation in the context of this design should facilitate successful discrimination of these compounds. By contrast, if dopamine functions to assign scalar value to antecedent cues, then pairing $\mathrm{X}$ with dopamine should simply increase X's value, facilitating its ability to serve as a conditioned reinforcer while interfering with its ability to support differential learning and responding in the two conditions.

Prior to training, all rats underwent surgery to infuse virus and implant fiber optics targeting the VTA as in Experiment 1 and 2 (Fig. 1; ChR2 experimental group, $n=6$; eYFP control group, $n$ $=6$ ). After surgery and recovery, rats were food deprived and then trained on the task. Configural training continued for 14 days, with rats receiving 40 trials each day. Rats received 10 trials of A without reward, and 10 trials where A was followed by 


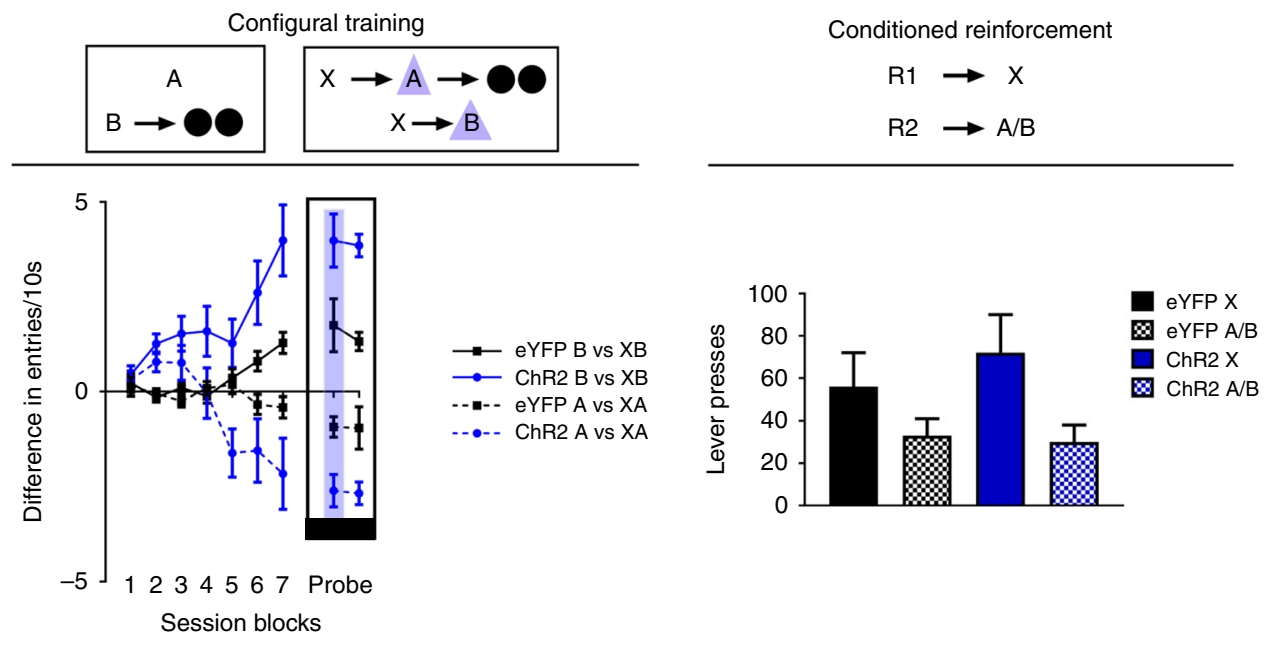

Fig. 4 Brief optogenetic stimulation of dopamine neurons facilitates configural learning without endowing cues with value. Left panel: Stimulation of dopamine neurons in the ChR2 group $(n=6)$ facilitates configural learning, relative to our eYFP control group $(n=6)$. A and B indicate auditory cues, X indicates a visual cue, and black circles show delivery of food pellets. VTA dopamine neurons were activated by light delivery (blue triangle) at the beginning of $A$ and $B$ only when preceeded by $X$. In a final session of configural training (Probe Test, outlined with black rectangle), we presented rats with a standard configural training session, with the exception that half the configural trials were presented with light (shadowed column in blue), and the other half were presented without light. For raw data during configural training, see Supplementary Fig. 5. Right panel: Stimulation of dopamine neurons during configural training does not produce changes in conditioned reinforcement. R1 and R2 indicate differently positioned levers, and rates of responding are represented as total number of lever presses within a session $( \pm S E M)$. For individual rats' responses during conditioned reinforcement testing, see Supplementary Fig. 6.

two 45 -mg sucrose pellets when immediately preceeded by $\mathrm{X}$ (i.e., $\mathrm{A} \rightarrow$ nothing; $\mathrm{X} \rightarrow \mathrm{A} \rightarrow 2 \mathrm{US}$ ). During these sessions, rats also received 10 trials where $B$ was presented with two 45-mg sucrose pellets, and 10 trials in which $\mathrm{B}$ was presented without reward when it was immediately preceded by $\mathrm{X}$ (i.e., $\mathrm{B} \rightarrow 2 \mathrm{US}$; $\mathrm{X} \rightarrow \mathrm{B} \rightarrow$ nothing). All four trial types were presented in an interleaved and pseudorandom manner, where no trial type could occur more than twice consecutively. On $\mathrm{X} \rightarrow \mathrm{A}$ and $\mathrm{X} \rightarrow \mathrm{B}$ trials, blue light $(473 \mathrm{~nm}, 20 \mathrm{~Hz}, 16-18 \mathrm{~mW}$ output; Shanghai Laser \& Optics Century Co., Ltd) was delivered for $1 \mathrm{~s}$ at the beginning of cue $\mathrm{A}$ and $\mathrm{B}$ to activate VTA dopamine neurons.

Across training, rats gradually learned to discriminate between the differently-rewarded trial types sharing a common cue (Fig. 4, configural training). The ability to discriminate these trial types emerged faster in rats in the ChR2 group, demonstrating that dopamine stimulation on the configural trials facilitated learning and did so both when the configural cue predicted reward and also when it did not. A three-factor ANOVA (cue $\times$ session $\times$ group; Fig. 4, top left) revealed a main effect of cue (i.e., AX-A vs. B-BX; $\left.F_{(1,10)}=7.567, p=0.020\right)$, a main of session $\left(F_{(6,60)}=\right.$ $7.574, p=0.000)$, and a session $\times$ group interaction $\left(F_{(6,60)}=\right.$ $2.442, p=0.035)$. The source of this interaction was due to rats in the ChR2 group showing faster acquisition of both configural discriminations (i.e., $\mathrm{AX}-\mathrm{A}$ and $\mathrm{X}-\mathrm{BX}$ ), revealing itself most prominently in the final session block with a significant betweengroup difference $\left(F_{(1,10)}=5.166, p=0.046\right)$. In addition, there was a significant cue $\times$ session $\times$ group interaction $\left(F_{(6,60)}=2.470\right.$, $p=0.034)$, owed to the faster $\mathrm{B}-\mathrm{XB}$ discrimination relative to XAA discrimination in ChR2 rats in early trials. However, rats in the ChR2 group showed significant effects of session in regards to both trial types (AX-X: $F_{(6,5)}=8.962, p=0.015$; B-BX: $F_{(6,5)}=$ $6.205, p=0.032$ ), that was not significant in eYFP rats (AX-X: $F_{(6,5)}=0.493, p=0.793$; B-BX: $\left.F_{(6,5)}=0.335, p=0.892\right)$. Importantly, these effects were not due to any real-time performance enhancing effect of dopamine, but instead reflected learning. This was evident in a final probe session at the end of configural training in which the laser was not activated on half the trials (Fig. 4, probe). During this test, we found no difference in responding to the cues depending on whether or not light was present as assessed by a three-factor ANOVA (light: $F_{(1,10)}=$ 0.077, $p=0.787$; light $\times$ group: $F_{(1,10)}=0.039, p=0.847$; cue $\times$ light: $F_{(1,10)}=0.445, \quad p=0.520 ; \quad$ cue $\times$ light $\times$ group: $\quad F_{(1,10)}=$ $0.071, p=0.795)$. Thus, activation of dopamine neurons after $\mathrm{X}$ enhanced learning to respond differently to the compound cues, suggesting that the dopamine transients facilitated acquisition of the "X-A" and "X-B" configural associations, rather than simply endowing $X$ with a high value.

To confirm this, the rats underwent conditioned reinforcement testing to assess whether value had accrued to $\mathrm{X}$, independent of the enhanced discrimination learning. Rats were tested in two sessions in which two levers were made available in the experimental chamber, one that led to a $2 \mathrm{~s}$ presentation of $\mathrm{X}$ and another that led to a $2 \mathrm{~s}$ presentation of either $\mathrm{A}$ or $\mathrm{B}(\mathrm{R} 1 \rightarrow \mathrm{X}$, $\mathrm{R} 2 \rightarrow \mathrm{A} / \mathrm{B}$, with the order of the $\mathrm{A}$ and $\mathrm{B}$ sessions counterbalanced across subjects in each group). By this time, the rats had received a total of 280 trials where X preceeded dopamine stimulation. If the artificial dopamine transient functioned as a teaching signal for caching value in antecedent cues, as predicted by model-free learning, then $\mathrm{X}$ should support robust conditioned reinforcement in the ChR2 group relative to the eYFP group. Yet we found no difference in the rates of lever pressing for $\mathrm{X}$ between groups (Fig. 4, conditioned reinforcement).

Interestingly, both groups did show higher responding to $\mathrm{X}$ relative to $\mathrm{A}$ or $\mathrm{B}$. There are two explanations for why this might occur. First, as X is a better predictor of $\mathrm{A}$ and $\mathrm{B}$, relative to $\mathrm{A}$ and $\mathrm{B}$ as predictors of reward, attention may be higher to $\mathrm{X}$, in line with a Mackintosh attentional mechanism, which predicts higher attentional capture for cues that predict future events 26,27 . Another possibility is that the visual cue $\mathrm{X}$ promoted greater orienting responses than the auditory A or B. As X was delivered from a light source at the top of the experimental chamber above the levers, it could drive the rats to be closer to the levers on $\mathrm{X}$ 
trials. In contrast, auditory cues $\mathrm{A}$ and $\mathrm{B}$ were diffuse around the chamber. Importantly, there was no difference in the magnitude of differential responding to $\mathrm{X}$ vs $\mathrm{A} / \mathrm{B}$ between groups (Fig. 4, conditioned reinforcement). A two-factor ANOVA (cue $\times$ group) revealed only a main effect of cue (cue: $F_{(1,10)}=9.608, p=0.011$; group: $F_{(1,10)}=0.205, p=0.660$; interaction: cue $\times$ group: $F_{(1,10)}$ $=0.829, p=0.384)$. And the lack of an effect of group was true regardless of whether the comparator for $\mathrm{X}$ was $\mathrm{A}$ or $\mathrm{B}$, revealed by a three-factor ANOVA (comparator: $F_{(1,10)}=0.014, p=0.907$; comparator x group: $F_{(1,10)}=0.120, p=0.737$; cue $\times$ comparator: $F_{(1,10)}=0.700, \quad p=0.422$; cue $\times$ comparator $\times$ group: $F_{(1,10)}=$ $0.514, p=0.490)$. Thus, even when a dopamine transient was delivered many times in a situation where rewards are available, we did not see evidence of value-caching, but rather we saw evidence for use of the signal to enhance value-independent associative learning.

\section{Discussion}

Here we have tested whether dopamine transients, like those observed in response to reward prediction errors, obey the predictions of model-free reinforcement learning accounts when placed into a context where external events are arranged such that prediction errors might be expected to occur. These theoretical accounts hold that dopaminergic prediction-error signals should result in value-caching and should not facilitate the formation of an associative model of events. Recent experiments have challenged these predictions, showing that artificial dopamine transients can support the formation of what appear to be associative representations ${ }^{6,8}$. However, these experiments did not directly assess value, independent of their measures of associative learning. This is important because dopamine transients could have two functions, one to support value learning and another to support learning of associative relationships. Or, as some critics have suggested, the apparent associative learning function in these studies may simply be a side effect of hidden or unappreciated value learning. If either of these explanations are correct, then measures of associative learning and value should covary. Contrary to this prediction, the current results show that dopamine signals, constrained within situations appropriate for associative learning, cause the acquisition of an associative model of the environment without evidence of value-caching.

Evidence that dopamine transients support associative learning, independent of value-caching, was shown in three experiments. In each experiment, the cues antecedent to the dopamine transient failed to show any evidence of value. In the first two experiments pairing neutral cues with dopamine stimulation did not allow these cues to support conditioned reinforcement. Specifically, rats would not press a lever to receive presentation of A or $\mathrm{C}$, showing that they did not become valuable to them. In a third experiment, we found that pairing reward-predictive cues with dopamine stimulation also did not alter their ability to produce conditioned reinforcement. That is, rats in the ChR2 group would not press more for X, relative to the eYFP group, even though this cue had been paired with dopamine in the context of reward learning. Importantly, in this experiment rats received a total of 280 trials with dopamine stimulation. If dopamine had endowed these cues with value, we would expect them to support conditioned reinforcement. In contrast to this hypothesis, we found no between-group difference in the ability of these cues to promote conditioned reinforcement. These data show that transient stimulation of dopamine during associative learning does not endow cues with model-free value.

At the same time, the dopamine transient facilitated the associative or model-based learning appropriate for these cues. Notably, this was true even when resultant behavior was the opposite of what would be predicted if the dopamine transient were being used for learning value. In the first experiment, the introduction of a dopamine transient at the beginning of $\mathrm{X}$ when it was preceded by $\mathrm{C}$ unblocked the association between $\mathrm{C}$ and $\mathrm{X}$. This unblocking was evident in the increase in food-port responding elicited by $\mathrm{C}$ after its associate, $\mathrm{X}$, was paired directly with reward. The second experiment extended this finding to show that the dopamine transient produces the same effect caused by overlearning of the cue pairs, previously seen both in other labs ${ }^{20-23}$ and in our hands (see Supplementary Fig. 3). Rather spectacularly, here the dopamine transient functioned to reduce a Pavlovian response directed towards a dopamine-paired cue, in opposition to a cached-value learning hypothesis. Finally, we also showed that introduction of a dopamine transient facilitates configural learning, in a manner that was again in opposition to a value hypothesis. Thus, these results add to the evidence that dopamine transients are sufficient to instantiate learning of associative representations of events and further show that the same transients do not necessarily function to attach a scalar value to those same events.

Interestingly, the same rats would work to directly activate the dopamine neurons (Supplementary Fig. 2), suggesting that our negative results are not due to poor viral expression, fiber placement or other factors that might impair activation of the dopamine neurons. In addition, this result shows, consistent with much other work, that dopamine delivered for longer time periods or in situations less structured or constrained to isolate associative learning can clearly produce a motivation and behavioral drive that could be called value ${ }^{7,28-33}$. In fact, it has recently been shown that directly pairing a cue with optogenetic activation of dopamine neurons, many times and without an obvious external precipitating event, can produce a cue that supports conditioned reinforcement ${ }^{28}$. Such results and our findings here are not mutually exclusive, rather they are complementary, revealing what the biological signal might do under different external conditions.

This point is illustrated by considering psychological disorders. People with schizophrenia show spurious or overly prominent dopaminergic prediction-error signals during learning ${ }^{34,35}$. Such signals, triggered by external events, might drive abnormal or inappropriate associative learning, contributing to the positive symptoms that characterize the disorder, with minimal impact on value learning. In contrast, addictive drugs cause dopamine to be released at times less precisely related to external events and for durations much longer than is typically observed in response to prediction errors. Such release might endow cues, contexts, or events that happen to be present with excessive value, contributing to uncontrollable drug-seeking, with minimal impact on associative representations ${ }^{36}$. These results stress that an understanding of the role of dopamine must consider the form of the signal and the context in which it occurs.

\section{Methods}

Surgical procedures. Rats received bilateral infusions of $1.2 \mu \mathrm{L}$ AAV5-EF1 $\alpha-D I O$ ChR2-eYFP $(n=28)$ or AAV5-EF1 $\alpha$-DIO-eYFP $(n=28)$ into the VTA at the following coordinates relative to bregma: AP: $-5.3 \mathrm{~mm}$; ML: $\pm 0.7 \mathrm{~mm}$; DV: -6.5 $\mathrm{mm}$ and -7.7 (females) or $-7.0 \mathrm{~mm}$ and $-8.2 \mathrm{~mm}$ (males). Virus was obtained from the Vector Core at University of North Carolina at Chapel Hill (UNC Vector Core). During surgery, optic fibers were implanted bilaterally $(200-\mu \mathrm{m}$ diameter, Thorlabs) at the following coordinates relative to bregma: AP: $-5.3 \mathrm{~mm}$; ML: \pm $2.61 \mathrm{~mm}$ and DV: $-7.05 \mathrm{~mm}$ (female) or $-7.55 \mathrm{~mm}$ (male) at an angle of $15^{\circ}$ pointed toward the midline. All procedures were conducted in accordance with the Institutional Animal Care and Use Committee of the US National Institutes of Health (approved protocol: 18-CNRB-108).

Apparatus. Training was conducted in eight standard behavioral chambers (Coulbourn Instruments; Allentown, PA), which were individually housed in lightand sound-attenuating boxes (Jim Garmon, JHU Psychology Machine Shop). Each 
chamber was equipped with a pellet dispenser that delivered 45-mg pellets into a recessed food port when activated. Access to the food port was detected by means of infrared detectors mounted across the opening of the recess. Two differently shaped panel lights were located on the right wall of the chamber above the food port. The chambers contained a speaker connected to white noise and tone generators and a relay that delivered a $5-\mathrm{kHz}$ click stimulus. A computer equipped with GS3 software (Coulbourn Instruments, Allentown, PA) controlled the equipment and recorded the responses. Raw data were output to and processed in Matlab (Mathworks, Natick, MA) to extract relevant response measures, which were analyzed in SPSS software (IBM analytics, Sydney, Australia).

Housing. Rats were housed singly and maintained on a 12-h light-dark cycle; all behavioral experiments took place during the light cycle. Rats had ad libitum access to food and water unless undergoing the behavioral experiment, during which they received either 8 grams or 12 grams of grain pellets- for females and males, respectively, daily in their home cage following training sessions. Rats were monitored to ensure they did not drop below $85 \%$ of their initial body weight across the course of the experiment. All experimental procedures were conducted in accordance with the NIDA-IRP Institutional Animal Care and Use Committee of the US National Institute of Health guidelines.

General behavioral procedures. Trials consisted of 10-s cues as described below. Trial types were interleaved in miniblocks, with the specific order unique to each rat but counterbalanced within each group. Inter-trial intervals varied around a 6 min mean. Unless otherwise noted, daily training was divided into a morning (AM) and afternoon (PM) session.

Response measures. We measured entry into the food port to assess conditioned responding. Food port entries were registered when the rat broke a light beam placed across the opening of the food port. This simple measure allowed us to calculate a variety of metrics including response latency after cue onset, number of entries to the food port during the cue, and the overall percentage of time spent in the food port during the cue. These metrics were generally correlated during conditioning, and all reflect to some extent the expectation of food delivery at the end of the cue in a task such as that used here. Generally when analyzing behavior during the sensory preconditioning task, we measure conditioned responding by calculating the percent of time rats spend in the food port during cue presentation $18,24,25$. The exception to this has been when analyzing behavior in the blocking of sensory preconditioning task. Specifically, in our previous manuscript we reported behavior in the blocking of sensory preconditioning procedure using number of entries into the food port during cue presentation ${ }^{6}$. However, in the current manuscript we have represented the data as the percent of time spent in the food port for two reasons. First, this allowed us to be consistent across experiments using the sensory preconditioning procedures within this manuscript. Second, we found that this particular cohort of rats exhibited lower levels of magazine entries into the food port than we have previously found using similar designs, likely reflecting subtle variations in the rats' environment and our experimental procedures across time. Importantly, both measures of behavior were highly correlated and elicited significant results in our critical probe tests (see Supplementary Fig. 1 for data as assessed by entries into the food port in Experiment 1, as well as additional analyses comparing the two response measures for this experiment).

Histology. All rats were euthanized with an overdose of carbon dioxide and perfused with phosphate buffered saline (PBS) followed by $4 \%$ paraformaldehyde (Santa Cruz Biotechnology Inc., CA). Fixed brains were cut in $40-\mu \mathrm{m}$ sections to examine fiber tip position and virus expression under a fluorescence microscope (Olympus Microscopy, Japan).

Statistical analyses. All statistics were conducted using the SPSS 24 IBM statistics package. Generally, analyses were conducted using a mixed-design repeatedmeasures ANOVA. All analyses of simple main effects were planned and orthogonal and therefore did not necessitate controlling for multiple comparisons. Data distribution was assumed to be normal, but homoscedasticity was not formally tested. Except for histological analysis, data collection and analyses were not performed blind to the conditions of the experiments.

Experiment 1. Sixteen experimentally naive male and female Long-Evans transgenic rats of $\sim 4$ months of age at surgery and carrying a TH-dependent Cre expressing system (NIDA Animals Breeding Facility) were used in this study. Sample sizes were chosen based on similar prior experiments that have elicited significant results with a similar number of rats. No formal power analyses were conducted. Rats were randomly assigned to groups and distributed equally by age, gender, and weight. Prior to final data analysis, four rats were removed from the experiment due to virus or cannula misplacement as verified by histological analysis.

The blocking of sensory preconditioning procedure was essentially identical to that used previously ${ }^{6}$. Training used a total of six different stimuli, drawn from stock equipment available from Coulbourn and included four auditory (tone, siren, clicker, white noise) and two visual stimuli (flashing light, steady light). Assignment of these stimuli to the cues depicted in Fig. 2 and described in the text was counterbalanced across rats in each group within each modality (A and $\mathrm{E}$ were visual while $\mathrm{C}, \mathrm{D}, \mathrm{F}$, and $\mathrm{X}$ were auditory).

Training began with $2 \mathrm{~d}$ of preconditioning. On the first day, the rats received 16 presentations of $\mathrm{A} \rightarrow \mathrm{X}$, in which a 10 -s presentation of $\mathrm{A}$ was immediately followed by a 10-s presentation of X. On the second day, the rats received eight presentations of $\mathrm{A} \rightarrow \mathrm{X}$ alone, as well as eight presentations each of three 10-s compound cues (EF, $\mathrm{AD}, \mathrm{AC}$ ) followed by $\mathrm{X}$ (i.e., $\mathrm{EF} \rightarrow \mathrm{X} ; \mathrm{AD} \rightarrow \mathrm{X} ; \mathrm{AC} \rightarrow \mathrm{X}$ ). On AC trials, light ( $473 \mathrm{~nm}, 16-18 \mathrm{~mW}$ output; Shanghai Laser \& Optics Century Co. Ltd) was delivered into the VTA for $1 \mathrm{~s}$ at a rate of $20 \mathrm{~Hz}$ at the beginning of X; on $\mathrm{AD}$ trials, the same light pattern was delivered during the intertrial interval, $120-180 \mathrm{~s}$ after termination of X. Following preconditioning, rats underwent $4 \mathrm{~d}$ of conditioning in which $\mathrm{X}$ was presented 24 times each day and was followed immediately by delivery of two $45-\mathrm{mg}$ sucrose pellets (5TUT; TestDiet, MO).

Following this training, rats received two different test sessions: a conditioned reinforcement test to assess value attribution to the preconditioned cues and a probe test to provide formal evidence of preconditioning. The order of these tests sessions was counterbalanced within the rats in each group such that half the rats in each group received the conditioned reinforcement test first, and the other half of the rats received the probe test first. On the following day, this order was reversed so that all rats received the alternate test. This pattern was then repeated once more so that all rats had two conditioned reinforcement tests and two probe tests. During conditioned reinforcement testing, the food ports were removed from the chamber and two levers were placed into the box for the first time, as in previous experiments ${ }^{17,18}$. Pressing one lever resulted in a $2 \mathrm{~s}$ presentation of cue $\mathrm{D}$, while pressing the other lever resulted in a $2 \mathrm{~s}$ presentation of cue $\mathrm{C}$. This session lasted for $30 \mathrm{~min}$. During the probe test, the chambers were kept as per the earlier training sessions and cues C and D were presented six times each in an interleaved and counterbalanced order, alone and without reward. Analyses were conducted on data overall sessions, where analyses for the probe tests were restricted to the last 5 $s$ of the first six trials of cue presentation in each probe session as done previously ${ }^{6}$

Following these test sessions, all rats received a final conditioned reinforcement test session. During this session, the two levers were put into the chamber and pressing one again resulted in a $2 \mathrm{~s}$ presentation of cue $\mathrm{D}$, while pressing the other resulted in a $2 \mathrm{~s}$ presentation of cue X. These sessions lasted for $30 \mathrm{~min}$. This provided a positive control to show that these rats would exhibit conditioned reinforcement for a cue directly paired with food using our procedures.

Experiment 2. Twenty-eight experimentally naive male and female Long-Evans transgenic rats of approximately 4 months of age at surgery and carrying a $\mathrm{TH}$ dependent Cre expressing system (NIDA animal breeding facility) were used in this study. Sample sizes were chosen based on similar prior experiments that elicited significant results with a similar number of rats. No formal power analyses were conducted. Rats were randomly assigned to groups and distributed equally by age, gender, and weight. Prior to final data analysis, five rats were removed from the experiment due to illness, virus or cannula misplacement.

For sensory preconditioning, we used a total of four different auditory stimuli, drawn from stock equipment available from Coulbourn, which included tone, siren, clicker, and white noise. Assignment of these stimuli to the cues depicted in Fig. 3 and described in the text was counterbalanced across rats. Training began with $1 \mathrm{~d}$ of preconditioning, in which where rats received 12 presentations of the $\mathrm{A} \rightarrow \mathrm{B}$ serial compound and 12 trials of the $\mathrm{C} \rightarrow \mathrm{D}$ serial compound. Following preconditioning, rats began conditioning, in which they received 24 trials of $B$ and 24 trials of D, where B was immediately followed by presentation of two $45-\mathrm{mg}$ sucrose pellets and $\mathrm{D}$ was presented in the absence of reward. Rats received 4 days of conditioning in this manner.

Following this training, rats again received a probe test to assess preconditioning and a conditioned reinforcement test to assess value attribution to the preconditioned cues. The order of these test sessions was again counterbalanced so that half the rats in each group received the conditioned reinforcement test first and the other half of the rats received the probe test first. During the probe test, the chambers were kept as per the earlier training sessions and cues A and C were presented six times each in an interleaved and counterbalanced order, alone and without reward. During the conditioned reinforcement test, the food ports were removed from the chamber and two levers were placed in the box for the first time, as done in previous experiments $\mathrm{s}^{17,18}$. Pressing one lever resulted in a $2 \mathrm{~s}$ presentation of cue $\mathrm{A}$, while pressing the other lever resulted in a $2 \mathrm{~s}$ presentation of cue C. These sessions lasted for $30 \mathrm{~min}$.

Following these test sessions, all rats received a final conditioned reinforcement test session. During this session, the two levers were put into the chamber and pressing one again resulted in a $2 \mathrm{~s}$ presentation of cue $\mathrm{B}$, while pressing the other resulted in a $2 \mathrm{~s}$ presentation of cue $\mathrm{D}$. These sessions lasted for $30 \mathrm{~min}$ and provided a positive control for the ability of thease rats to show conditioned reinforcement under our procedures.

Experiment 3. Twelve experimentally naive male and female Long-Evans transgenic rats of $\sim 4$ months of age at surgery and carrying a TH-dependent Cre expressing system (NIDA animal breeding facility) were used in this study. Sample sizes were chosen based on similar prior experiments that elicited significant results 
with a similar number of rats. No formal power analyses were conducted. Rats were randomly assigned to groups and distributed equally by age, gender, and weight. All rats were included in the final analyses.

For configural training, we used a total of three different stimuli, a flashing light controlled by Coulbourn, and a chime and siren sound produced by Arduino software. Assignment of these stimuli to the cues depicted in Fig. 4 and described in the text was counterbalanced across rats (X was visual, while $\mathrm{A}$ and $\mathrm{B}$ were auditory). Configural training consisted of four different trial types within the same session, randomly presented, where no trial type could occur more than twice consecutively. On elemental trial types, A was presented without food, while B was presented and followed immediately by delivery of two 45-mg sucrose pellets. On configural trials, $\mathrm{X}$ was presented immediately before $\mathrm{A}$ and $\mathrm{B}$ to form two serial compounds $\mathrm{X} \rightarrow \mathrm{A}$ and $\mathrm{X} \rightarrow \mathrm{B}$. In contrast to elemental trials, $\mathrm{X} \rightarrow \mathrm{A}$ was followed immediately by two 45 -mg sucrose pellets, while $\mathrm{X} \rightarrow \mathrm{B}$ was presented in the absence of reward. Rats received 14 days of training, receiving one session consisting of 40 trials per day. In a final session, we gave rats a standard training session with the exception that light was omitted on half the trails. All other aspects of the session remained the same as during training.

Following this training, rats received two conditioned reinforcement tests. During these tests, the food ports were removed from the chamber and two levers were placed in the box for the first time, as done in previous experiments. In one conditioned reinforcement test, pressing one lever resulted in a $2 \mathrm{~s}$ presentation of $\mathrm{X}$, while pressing the other resulted in a $2 \mathrm{~s}$ presentation of $\mathrm{A}$. In the other conditioned reinforcement test, pressing one lever continued to result in a $2 \mathrm{~s}$ presentation of $\mathrm{X}$, while pressing the other resulted in a $2 \mathrm{~s}$ presentation of $\mathrm{B}$. The order in which rats received these sessions was fully counterbalanced. These sessions lasted for $30 \mathrm{~min}$

Reporting summary. Further information on research design is available in the Nature Research Reporting Summary linked to this article.

\section{Data availability}

The data that support the findings of this study, and any associated custom programs used for its acquisition, are available from the corresponding authors upon reasonable request.

Received: 3 August 2019; Accepted: 5 December 2019; Published online: 08 January 2020

\section{References}

1. Mirenowicz, J. \& Schultz, W. Importance of unpredictability for reward responses in primate dopamine neurons. J. Neurophysiol. 72, 1024-1027 (1994).

2. Schultz, W., Dayan, P. \& Montague, P. R. A neural substrate for prediction and reward. Science 275, 1593-1599 (1997)

3. Sutton, R. S. \& Barto, A. G. Toward a modern theory of adaptive networks: expectation and prediction. Psychological Rev. 88, 135-170 (1981).

4. Sutton, R. S. \& Barto, A. G. Reinforcement learning: An introduction. (MIT press, Cambridge, 1998).

5. Schultz, W. Dopamine reward prediction-error signalling: a two-component response. Nat. Rev. Neurosci. 17, 183-195 (2016).

6. Sharpe, M. J. et al. Dopamine transients are sufficient and necessary for acquisition of model-based associations. Nat. Neurosci. 20, 735-742 (2017).

7. Tsai, H. C. et al. Phasic firing in dopamine neurons is sufficient for behavioral conditioning. Science 324, 1080-1084 (2009).

8. Keiflin, R., Pribut, H. J., Shah, N. B. \& Janak, P. H. Ventral tegmental dopamine neurons participate in reward identity predictions. Curr. Biol. 29, 93-103 (2019).

9. Adamantidis, A. R. et al. Optogenetic interrogation of dopaminergic modulation of the multiple phases of reward-seeking behavior. J. Neurosci. 31, 10829-10835 (2011).

10. Popescu, A. T., Zhou, M. R. \& Poo, M.-M. Phasic dopamine release in the medial prefrontal cortex enhances stimulus discrimination. Proc. Natl Acad. Sci. USA 113, E3169-E3176 (2016).

11. Chang, C. Y., Gardner, M., Di Tillio, M. G. \& Schoenbaum, G. Optogenetic blockade of dopamine transients prevents learning induced by changes in reward features. Curr. Biol. 27, 3480-3486.e3483 (2017).

12. Chang, C. Y. et al. Brief optogenetic inhibition of dopamine neurons mimics endogenous negative reward prediction errors. Nat. Neurosci. 19, 111-116 (2016).

13. Hamid, A. A. et al. Mesolimbic dopamine signals the value of work. Nat. Neurosci. 19, 117-126 (2016)
14. Witten, I. B. et al. Recombinase-driver rat lines: tools, techniques, and optogenetic application to dopamine-mediated reinforcement. Neuron $\mathbf{7 2}$, 721-733 (2011)

15. Cardinal, R. N., Parkinson, J. A., Hall, G. \& Everitt, B. J. Emotion and motivation: the role of the amygdala, ventral striatum, and prefrontal cortex. Neurosci. Biobehav. Rev. 26, 321-352 (2002).

16. Parkinson, J., Roberts, A., Everitt, B. \& Di Ciano, P. Acquisition of instrumental conditioned reinforcement is resistant to the devaluation of the unconditioned stimulus. Q. J. Exp. Psychol. Sect. B 58, 19-30 (2005).

17. Burke, K. A., Franz, T. M., Miller, D. N. \& Schoenbaum, G. The role of the orbitofrontal cortex in the pursuit of happiness and more specific rewards. Nature 454, 340-344 (2008)

18. Sharpe, M. J., Batchelor, H. M. \& Schoenbaum, G. Preconditioned cues have no value. elife 6, e28362 (2017)

19. Brogden, W. J. Sensory pre-conditioning. J. Exp. Psychol. 25, 323-332 (1939)

20. Holland, P. C. \& Ross, R. T. Savings test for associations between neutral stimuli. Anim. Learn. Behav. 11, 83-90 (1983).

21. Holland, P. C. Second-order conditioning with and without unconditioned stimulus presentation. J. Exp. Psychol. Anim. Behav. Process. 6, 238-250 (1980).

22. Forbes, D. T. \& Holland, P. C. Spontaneous configuring in conditioned flavor aversion. J. Exp. Psychol. Anim. Behav. Process. 11, 224-240 (1985).

23. Hoffeld, D. R., Kendall, S. B., Thompson, R. F. \& Brogden, W. Effect of amount of preconditioning training upon the magnitude of sensory preconditioning. J. Exp. Psychol. 59, 198-204 (1960).

24. Sadacca, B. F., Jones, J. L. \& Schoenbaum, G. Midbrain dopamine neurons compute inferred and cached value prediction errors in a common framework. elife 5, e13665 (2016).

25. Jones, J. L. et al. Orbitofrontal cortex supports behavior and learning using inferred but not cached values. Science 338, 953-956 (2012).

26. Le Pelley, M. E., Beesley, T. \& Griffiths, O. Overt attention and predictiveness in human contingency learning. J. Exp. Psychol. Anim. Behav. Process. 37, 220-229 (2011).

27. Mackintosh, N. J. A theory of attention: variations in the associability of stimuli with reinforcement. Psychological Rev. 82, 276-298 (1975).

28. Saunders, B., Richard, J., Margolis, E. \& Janak, P. Dopamine neurons create Pavlovian conditioned stimuli with circuit-defined motivational properties. Nat. Neurosci. 21, 1072-1083 (2017).

29. Wang, D. V. et al. Disrupting glutamate co-transmission does not affect acquisition of conditioned behavior reinforced by dopamine neuron activation. Cell Rep. 18, 2584-2591 (2017).

30. Arvanitogiannis, A. \& Shizgal, P. The reinforcement mountain: allocation of behavior as a function of the rate and intensity of rewarding brain stimulation. Behav. Neurosci. 122, 1126-1138 (2008).

31. Cheer, J. F. et al. Coordinated accumbal dopamine release and neural activity drive goal-directed behavior. Neuron 54, 237-244 (2007).

32. Scardochio, T., Trujillo-Pisanty, I., Conover, K., Shizgal, P. \& Clarke, P. B. The effects of electrical and optical stimulation of midbrain dopaminergic neurons on rat $50-\mathrm{kHz}$ ultrasonic vocalizations. Front. Behav. Neurosci. 9, 331 (2015).

33. Phillips, P. E., Stuber, G. D., Heien, M. L., Wightman, R. M. \& Carelli, R. M. Subsecond dopamine release promotes cocaine seeking. Nature 422, 614-618 (2003).

34. Corlett, P. R. et al. Disrupted prediction-error signal in psychosis: evidence for an associative account of delusions. Brain 130, 2387-2400 (2007).

35. Kapur, S. Psychosis as a state of aberrant salience: a framework linking biology, phenomenology, and pharmacology in schizophrenia. Am. J. Psychiatry 160, 13-23 (2003).

36. Everitt, B. J. \& Robbins, T. W. Neural systems of reinforcement for drug addiction: from actions to habits to compulsion. Nat. Neurosci. 8, 1481-1489 (2005).

\section{Acknowledgements}

The authors thank M.Morales, C Mejias-Aponte, and the NIDA histology core for their assistance. This work was supported by a C.J.Martin Fellowship awarded (to M.J.S.), and the Intramural Research Program at NIDA ZIA-DA000587 (to G.S.). The opinions expressed in this article are the authors' own and do not reflect the view of the NIH/ DHHS.

\section{Author contributions}

M.J.S. and G.S. designed the experiments; M.J.S., H.M.B., L.E.M, E.J.P., and C.Y.C. collected the data. M.J.S. analyzed the data. M.J.S., Y.N., and G.S. interpreted the data and wrote the manuscript with input from all authors.

\section{Competing interests}

The authors declare no competing interests. 


\section{Additional information}

Supplementary information is available for this paper at https://doi.org/10.1038/s41467019-13953-1.

Correspondence and requests for materials should be addressed to G.S.

Peer review information Nature Communications thanks the anonymous reviewers for their contribution to the peer review of this work.

Reprints and permission information is available at http://www.nature.com/reprints

Publisher's note Springer Nature remains neutral with regard to jurisdictional claims in published maps and institutional affiliations. (c) (i) Open Access This article is licensed under a Creative Commons Attribution 4.0 International License, which permits use, sharing, adaptation, distribution and reproduction in any medium or format, as long as you give appropriate credit to the original author(s) and the source, provide a link to the Creative Commons license, and indicate if changes were made. The images or other third party material in this article are included in the article's Creative Commons license, unless indicated otherwise in a credit line to the material. If material is not included in the article's Creative Commons license and your intended use is not permitted by statutory regulation or exceeds the permitted use, you will need to obtain permission directly from the copyright holder. To view a copy of this license, visit http://creativecommons.org/ licenses/by/4.0/.

(C) The Author(s) 2020 